\title{
CONTACT RESISTANCE OF InGaN/GaN LIGHT EMITTING DIODES GROWN ON THE PRODUCTION MODEL MULTI-WAFER MOVPE REACTOR
}

\author{
R.W. Chuang, A.Q. Zou, and H.P. Lee \\ Dept. of Electrical and Computer Engineering, University of California, Irvine, CA 92697 \\ Z.J. Dong, F.F. Xiong, and R. Shih \\ Alpha Photonics Inc., 2019 Saturn Street, Monterey Park, CA 91754 \\ M. Bremser \\ AIXTRON Inc., 1670 Barclay Blvd., Buffalo Grove, IL, 60089 USA \\ H. Juergensen \\ AIXTRON AG, 15-17 Kackertstrasse, Aachen D-52072 Germany
}

Cite this article as: MRS Internet J. Nitride Semicond. Res. 4S1, G6.42(1999)

\begin{abstract}
We report both the device fabrication and characterization of InGaN/GaN single quantum well LEDs grown on sapphire substrates using multi-wafer MOVPE reactor. To improve current spreading of the LEDs, a self-aligned process is developed to define LED mesa that is coated with a thin, semi-transparent $\mathrm{Ni} / \mathrm{Au}(40 \AA / 40 \AA)$ layer. A detailed study on the ohmic contact resistance of $\mathrm{Ni} / \mathrm{Cr} / \mathrm{Au}$ on $p$-GaN versus annealing temperatures is carried out on transmission line test structures. It was found that the annealing temperatures between 300 to $500{ }^{\circ} \mathrm{C}$ yield the lowest specific contact resistance $r_{c}\left(0.016 \Omega-\mathrm{cm}^{2}\right.$ at a current density of $66.7 \mathrm{~mA} / \mathrm{cm})$. Based on the extracted $r_{c}$ from the transmission line measurement, we estimate that the contact resistance of the $p$-type $\mathrm{GaN}$ accounts for $\sim 88 \%$ of the total series resistance of the LED.
\end{abstract}

\section{INTRODUCTION}

The III-V nitride semiconductor materials system has become increasingly popular among the applications of UV/blue light emitting diodes (LEDs), laser diodes (LDs) and hightemperature electronic devices due to its large direct bandgap energy ( $3.4 \mathrm{eV}$ for $\mathrm{GaN}$ at room temperature). Even though the potentials of III-V nitrides were well known for more than three decade, it was only until 1992 with the realization of $\mathrm{Mg}$ doped $p$-GaN film after the thermal annealing [1] could the realization of high brightness blue/green InGaN/GaN quantum well(s) LED [2] and the recent demonstrations of long-lifetime pulsed and CW blue LDs [3] be achieved. Despite the commercialization of LEDs, the low $p$-type doping concentration (and hence high $p$-type resistivity) in GaN still limits the device performance in two ways: first it reduces the current spreading in the LED. This causes most of the light to emit in the active region directly underneath the $p$-type contact, and is therefore blocked by the $p$ contact. Secondly, low $p$-type doping results in a rather high and non-linear contact resistance. While the current spreading problem has been effectively resolved by depositing a thin semitransparent metal layer on top of the $p-\mathrm{GaN}$ cap layer, high contact resistance is still a problem to be tackled. In the present work, we report the successful growth of single quantum well 
InGaN/GaN LEDs on multi-wafer MOVPE reactor with high thickness uniformity and the development of a self-aligned LED fabrication process that incorporates a thin current spreading layer. We also present detailed studies on the variation of contact resistance $r_{c}$ of $\mathrm{Ni} / \mathrm{Cr} / \mathrm{Au}$ on $p$-doped $\mathrm{GaN}$ layer versus annealing temperature.

\section{EXPERIMENT}

The InGaN/GaN LED samples were first grown on the Aixtron production model multi-wafer MOVPE reactor. The structures consist of $1.5 \mu \mathrm{m} n$-GaN layer, a thin InGaN quantum well (4-8 nm), and a $0.5 \mu \mathrm{m} p$-GaN cap layer, all grown on 2-inch (0001) sapphire substrates. The growth uses standard precursors including TMGa and TMIn for Group III and $\mathrm{NH}_{3}$ for Group $\mathrm{V}$ elements, and silane and $\mathrm{MgCp}_{2}$ are used for $\mathrm{n}$-type and p-type dopant sources, respectively. The un-intentionally doped $\mathrm{GaN}$ is $n$-type with carrier concentration $\sim 1$ $3 \times 10^{17} / \mathrm{cm}^{3}$. The $p$-type $\mathrm{GaN}$ with hole concentration of $\sim 5 \times 10^{17} / \mathrm{cm}^{3}$ are routinely achieved after post-growth annealing. The thickness uniformity across the diameter of a 2-inch wafer was evaluated using spectral reflectometry measurement in the range of 670 to $1100 \mathrm{~nm}$.

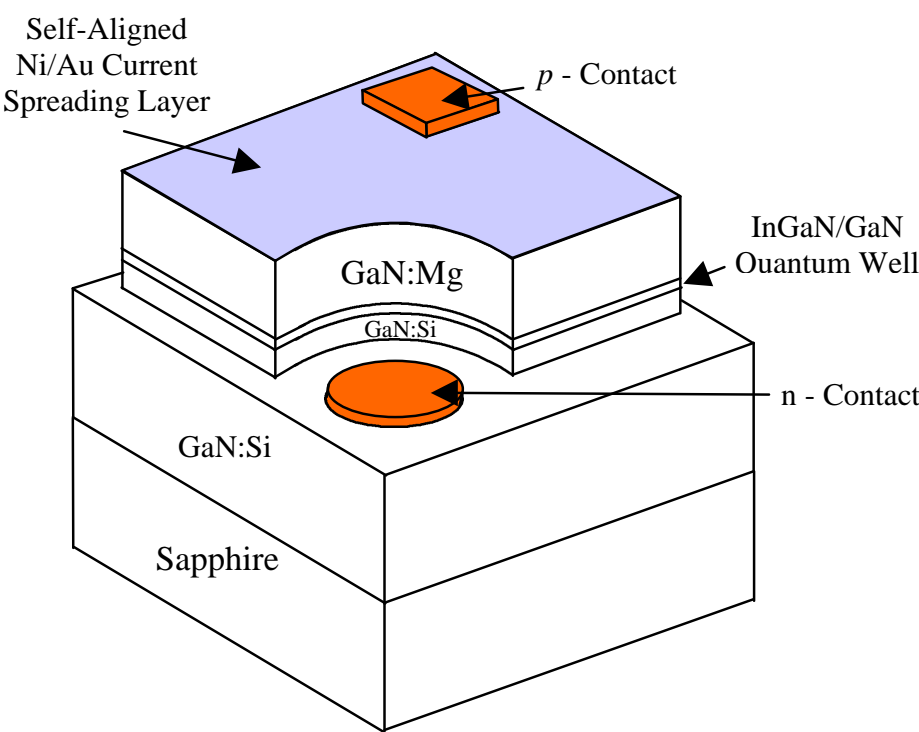

Fig. 1(a) The schematics of self-aligned InGaN/GaN LED structure. Note that a thin $\mathrm{Ni} / \mathrm{Au}$ layer is deposited on top of the LED mesa to improve the current spreading.

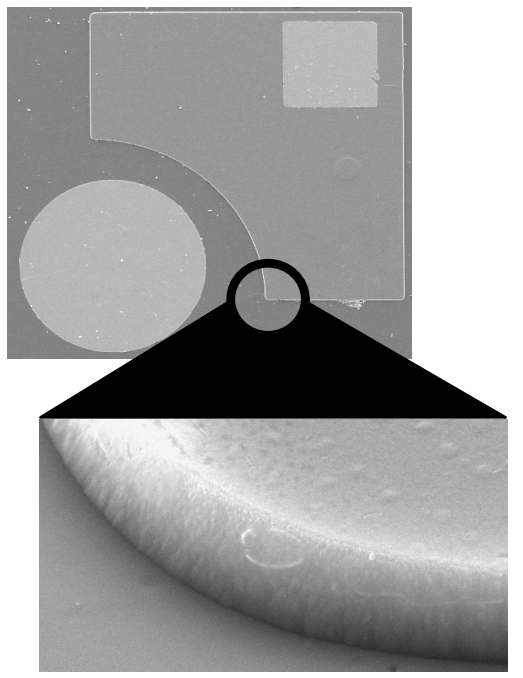

Fig. 1(b) SEM of a typical LED structure with a magnified view of the edges of mesa after RIE etching.

The process flow for fabricating a LED with a structure shown in Fig. 1(a), is described as follows. First, a thin current spreading layer consists of Ni/Au (40 $\mathrm{\AA} / 40 \AA)$ was deposited on the entire wafer using electron beam evaporation. The estimated optical absorption due to the current spreading layer is $\sim 38 \%$ [4]. The sample is thermally annealed at $500^{\circ} \mathrm{C}$ under $\mathrm{N}_{2}$ ambient for 100 seconds. The LED mesa is then defined by a photoresist pattern. This is followed by ion milling to remove all except the current spreading layer, and Reactive Ion Etching (RIE) in a mixture of $\mathrm{Cl}_{2}$ and $\mathrm{BCl}_{3}$ to remove the $\mathrm{GaN}$ all the way to the $n$-layer. In this way, the current spreading layer and the mesa are formed in a self-aligned way. Figure 1(b) shows the SEM micrographs of the RIE etched surface around the LED mesa. Both the 
bottom and lateral surfaces of the etched mesa appear to be very smooth. Finally, Ni/Cr/Au $(150 \AA / 150 \AA / 1200 \AA)$ were e-beam evaporated to form both $n$ and $p$-type contact pads. LEDs without current spreading layer are also fabricated for comparison. By varying the In composition and the thickness of the InGaN quantum well, LED emission from 420 to $490 \mathrm{~nm}$ were obtained.

To investigate the contact resistance to $p$-type $\mathrm{GaN}$, a rectangular shaped, mesa isolated transmission line (TL) structure was fabricated. The width of the TL pattern is $300 \mu \mathrm{m}$ while the spacing between adjacent $\mathrm{Ni} / \mathrm{Cr} / \mathrm{Au}$ contacts are varied from 5 to $50 \mu \mathrm{m}$ at increments of 5 $\mu \mathrm{m}$. The TL pattern is defined using RIE etching similar to the LED mesa etching. The wafer was then diced into several pieces each containing 4 TL patterns and was subsequently annealed at temperatures from $300^{\circ} \mathrm{C}$ to $600^{\circ} \mathrm{C}$ for 100 seconds. The TL structures are measured at constant current and the specific contact resistance $r_{c}$ 's were extracted using standard technique [5]. These experimental results will be discussed in the next section.

\section{RESULTS AND DISCUSSIONS}

Figure 2 shows a typical reflectance spectrum and the thickness nonuniformity across the diameter of a 2 -inch LED sample. The thickness of $n$-GaN layer for this particular LED sample is $\sim 3 \mu \mathrm{m}$. The epilayer thickness, $d$ is calculated from $d=\left[\lambda^{2} /(2 n \Delta \lambda)\right]$, where $\Delta \lambda$ is the wavelength separation between successive reflectance peak, and $\mathrm{n}$ is the refractive index of the GaN epilayer. The thickness shown in Fig. 2 are averaged using $\Delta \lambda$ from 5 successive reflectance peaks. Thickness nonuniformity of less than $<1 \%$ is observed across the entire 2inch wafer. The uniformity result we obtained on a typical LED device layer agrees reasonably well with Aixtron's own evaluation on 2-inch single-layer GaN wafer [6]. Figure 3(a) shows the current vs. voltage of the same LED wafer with and without the addition of the current spreading layer. It can be seen that the incorporation of current spreading layer has drastically reduced the spreading series resistance of the LED, which is caused by the high resistive $p$ $\mathrm{GaN}$ cap layer. With the current spreading layer, the operating voltage of the LED at $20 \mathrm{~mA}$ is reduced to $5 \mathrm{~V}$ or below. The use of self-alignment process for forming both the mesa and
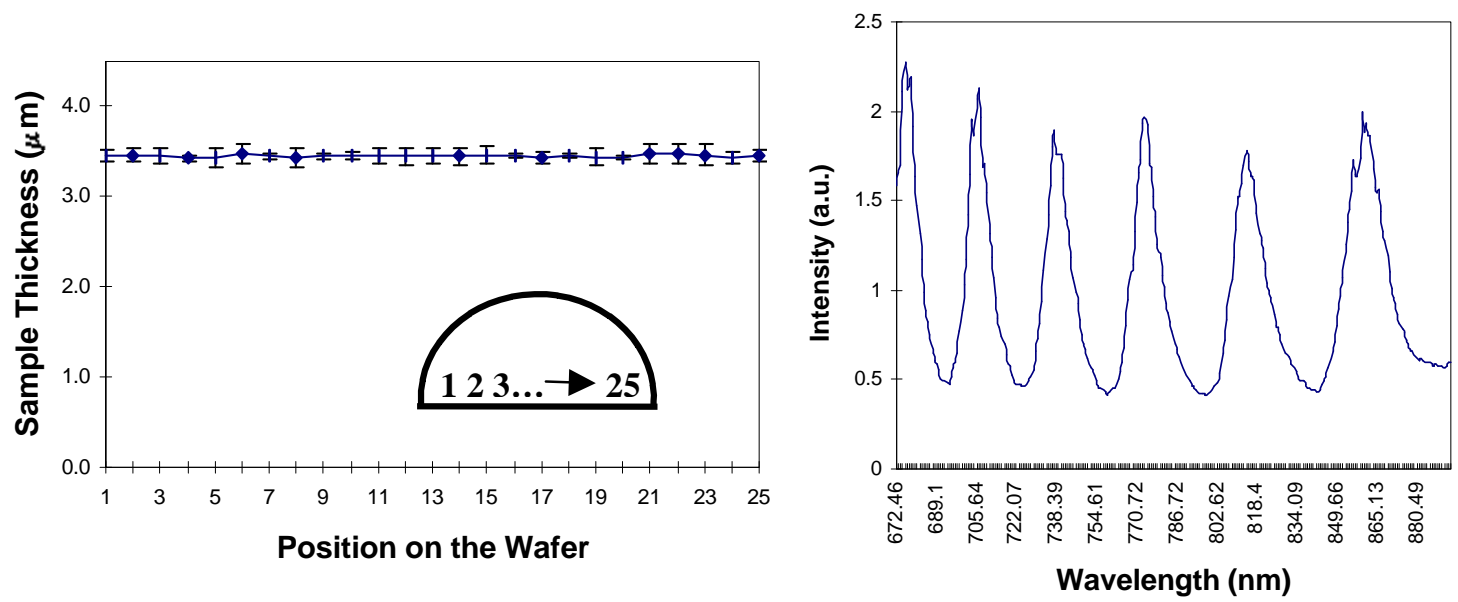

Fig. 2 Thickness uniformity across the diameter of a 2-inch LED wafer. A typical reflectance spectrum is also shown here. 
current spreading layer ensures maximum current spreading of a LED. Microscope observation shows that uniform emission from the entire LED mesa except the $p$-contact pad is achieved. The measured series resistance of $9.19 \times 10^{4} \mu \mathrm{m}^{2}$ LED is $\sim 26 \Omega$. Figure 3(b) shows the emission spectrum of a typical LED we fabricated. For this particular device, the emission peak is $\sim 490 \mathrm{~nm}$ and the FWHM is $\sim 50 \mathrm{~nm}$.

Owing to the low carrier concentration of the $p$-type $\mathrm{GaN}$ and the relative large barrier height between the metal and $p-\mathrm{GaN}$, the I-V characteristics of the TL are non-linear. That is, the contact resistance depends on the measurement current. Figure 4 shows a typical resistance vs. contact separation at two current levels. Figure 5 shows the extracted $r_{c}$ for $\mathrm{Ni} / \mathrm{Cr} / \mathrm{Au}$ system at different measurement currents for samples annealed at different temperatures. In all cases, the value of $r_{c}$ decreases monotonically with increasing current density (current/unit length) in the TL pattern and then approaches a saturation value. The specific contact resistance, $r_{c}$ is seen to decrease monotonically with increasing current. The optimal annealing temperature is observed to be $300-500^{\circ} \mathrm{C}$. Further increase in the annealing temperature at $600^{\circ} \mathrm{C}$ and above results in an increase of $r_{c}$. This phenomenon is likely caused by the formation of nitrogen vacancy that occurs when samples are subjected to high temperature treatments. The nitrogen vacancy in turn compensates the acceptor dopants at the metal $/ p-\mathrm{GaN}$ interface. Several groups have already reported similar findings on these issues $[7,8,9]$. In addition, the presence of the high resistive $\mathrm{Ni}_{3} \mathrm{~N}$ and $\mathrm{Ni}_{4} \mathrm{~N}$ phases at the metal-semiconductor interface at the annealing temperature greater than $500{ }^{\circ} \mathrm{C}$ can also result in higher contact resistance [10]. The $r_{c}$ measurement results are summarized in Table I. The lowest $r_{c}$ we obtained is about 0.016 $\Omega-\mathrm{cm}^{2}$ (the resistivity of the TL sample extracted is on the average of $4.05 \pm 0.15 \Omega-\mathrm{cm}$ ). The values $r_{c}$ we obtained are similar to previously reported data on $\mathrm{Ni} / p-\mathrm{GaN}$ system $[11,12]$. The lowering of $r_{c}$ due to thermal annealing below $600{ }^{\circ} \mathrm{C}$ is not clear at this stage. Earlier studies had suggested that thermal annealing of $\mathrm{Ni} / p-\mathrm{GaN}$ helps to removes the contaminants at the

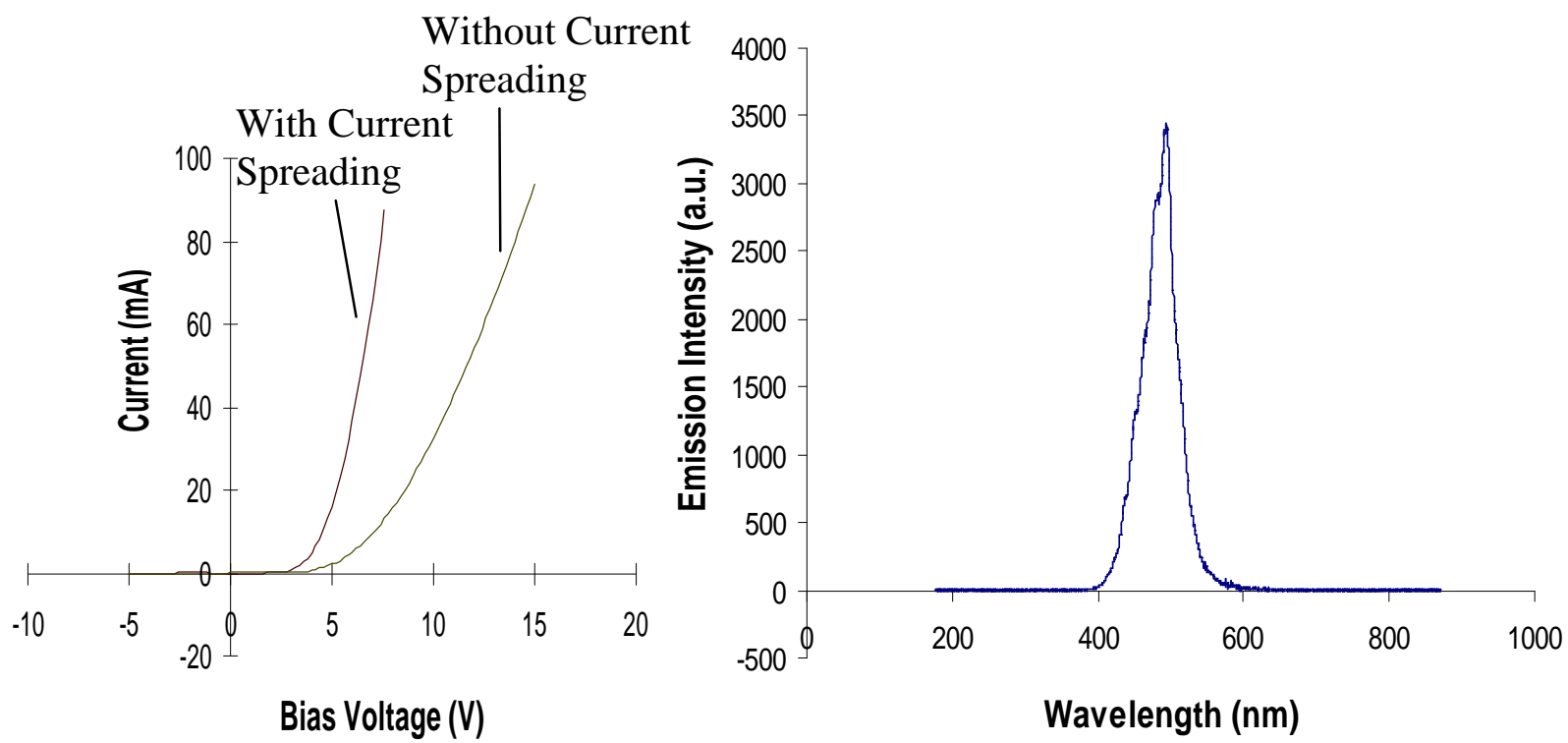

Fig 3 (a) The I-V of the LED with and without the current spreading layer; and (b) the emission spectrum of the LED. 
interface resulting in lowering of $r_{c}$ [12]. Recent results on the lowering of $r_{c}$ by aqua regia etching of $p$-GaN has strongly suggested that surface oxide plays a paramount role on the specific contact resistance to $p$-type GaN [13].

From the LED I-V, the extracted series resistance is $26 \Omega$ measured between 40 to 80 $\mathrm{mA}$. The current density of the LED operating at $60 \mathrm{~mA}$ corresponds to an average current density of $\sim 31.3 \mathrm{~mA} / \mathrm{cm}$ for the TL measurement. From Fig. 5, the corresponding $r_{c}$ extracted from the TL measurement is $0.021 \Omega-\mathrm{cm}^{2}$. The estimated contact resistance of the LED is therefore $\sim 22.9 \Omega$. Based on this estimate, over $88 \%$ of the series resistance of the LED

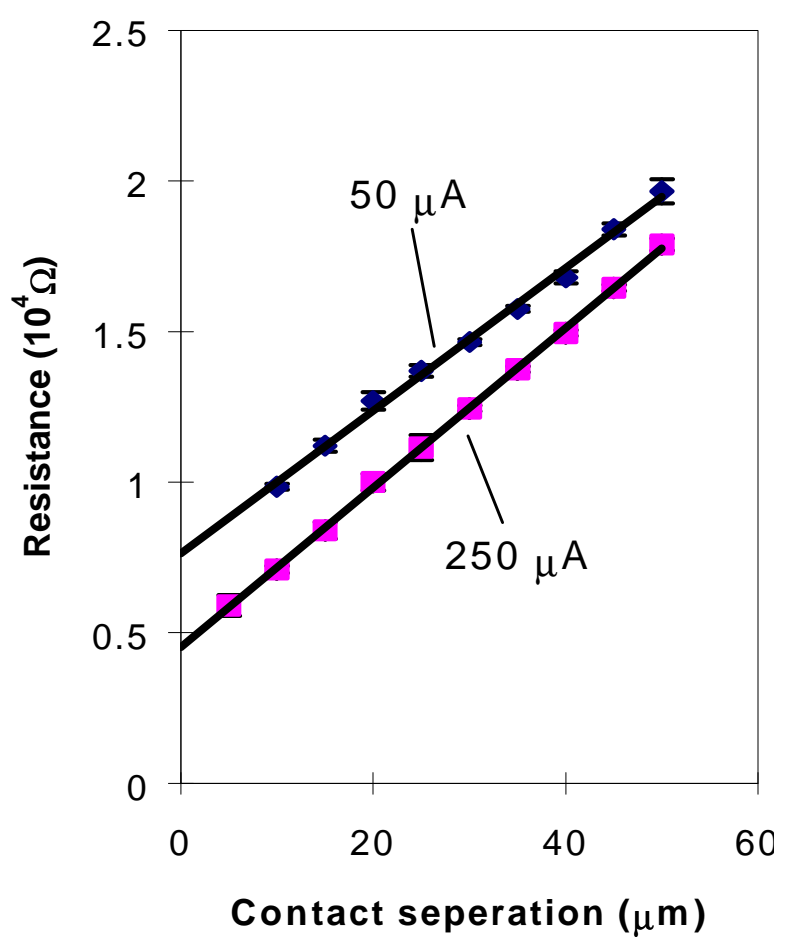

Fig. 4 The measured resistance versus spacing of the TL test pattern at two different measurement currents.

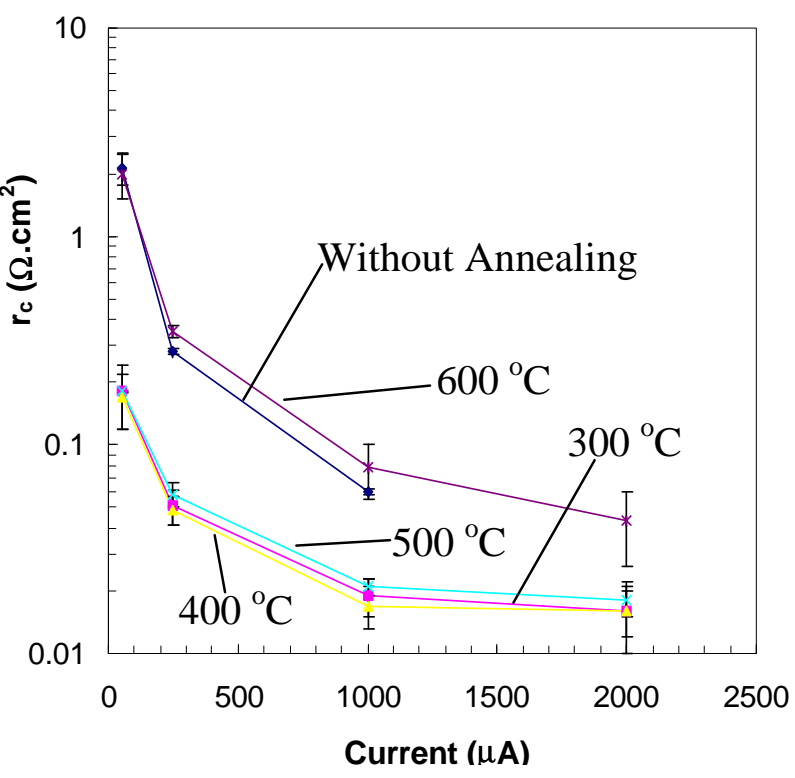

Fig. 5 The extracted $r_{c}$ measured at different current levels and annealing temperatures. The width of the rectangular TL pattern is $300 \mu \mathrm{m}$.

\begin{tabular}{|c|c|c|c|c|c|c|c|c|c|c|c|}
\hline & \multicolumn{10}{|c|}{ Specific Contact Resistance $(\Omega$ - Sqr. Cm) } \\
\hline Current $(\mu$ A $)$ & No Annealing & Std. Dev. & 300 Deg. C & Std. Dev. & 400 Deg. C & Std. Dev. & 500 Deg. C & Std. Dev & 600 Deg. C & Std. Dev \\
\hline 50 & 2.13 & 0.38 & 0.18 & 0.06 & 0.17 & 0.05 & 0.18 & 0.012 & 1.98 & 0.47 \\
\hline 250 & 0.28 & 0.01 & 0.051 & 0.01 & 0.049 & 0.007 & 0.058 & 0.008 & 0.34 & 0.024 \\
\hline 1000 & 0.06 & 0.002 & 0.019 & 0.004 & 0.017 & 0.004 & 0.021 & 0.002 & 0.078 & 0.023 \\
\hline 2000 & & & 0.016 & 0.006 & 0.016 & 0.004 & 0.018 & 0.004 & 0.043 & 0.017 \\
\hline
\end{tabular}

Table I The extracted average $r_{c}$ and the corresponding standard deviations from the TL test pattern at different current levels for samples under different annealing temperatures. 
originates from the $p$-GaN contact resistance.

\section{CONCLUSION}

We report highly uniform growth (thickness nonuniformity $<1 \%$ ) of InGaN/GaN LED on 2-inch sapphire substrate using a multi-wafer MOVPE reactor. A self-aligned LED fabrication process is developed to incorporate a thin $\mathrm{Ni} / \mathrm{Au}$ metal for improved current spreading of the LED device. It is also found that the annealing temperatures between 300-500 ${ }^{\circ} \mathrm{C}$ significantly reduce the contact resistance of as-deposited $\mathrm{Ni} / \mathrm{Cr} / \mathrm{Au}$ metals on $p$-type $\mathrm{GaN}$.

Specific contact resistance in the range of 0.016 to $0.021 \Omega-\mathrm{cm}^{2}$ is achieved at annealing temperatures between $300^{\circ} \mathrm{C}$ to $500^{\circ} \mathrm{C}$.

\section{REFERENCE}

1. S. Nakamura, N. Iwasa, M. Senoh, and T. Mukai, Jpn J. Appl. Phys., 31, 1258, (1992).

2. S. Nakamura, T. Mukai, and M. Senoh, Appl. Phys. Lett., 64, 1678, (1994).

3. S. Nakamura, M. Senoh, S. Nagahama, N. Naruhito, T. Yamada, T. Matsuchita, Y. Sugimoto, and H. Kiyoku, Appl. Phys. Lett., 69, 1477, (1996).

4. Handbook on Optical Constants of Solids Vol. I, II, and III, edited by D. Palik, Academic Press, (1998).

5. H. Murrmann and D. Widmann, IEEE Tran. Electron Device., 16, 1022-1024, (1969).

6. R. Beccard, O. Schoen, B. Schineller, D. Schmitz, M. Heuken, and H. Juergensen, Mat. Res. Soc. Symp. Proc., Vol. 482, 155, (1998).

7. L.F. Lester, J.M. Brown, J.C. Ramer, L. Zhang, S.D. Hersee, and J.C. Zolper, Appl. Phys Lett., 69, 2737, (1996).

8. J.C. Zolper, D.J. Rieger, A.G. Baca, S.J. Pearton, J.W. Lee, and R.A. Stall, Appl. Phys. Lett., 69, 538, (1996).

9. H.S. Venugopalan, S.E. Mohney, B.P. Luther, S.D. Wolter, and J.M. Redwing, J. Appl. Phys., 82(2), 15 July, 650, (1997).

10. J.K. Sheu, Y.K. Su, G.C. Chi, W.C. Chen, C.Y. Chen, C.N. Huang, J.M. Hong, Y.C. Yu, C.W. Wang, and E.K. Lin, J. Appl. Phys., 83(6), 15 March, 3172, (1998).

11. T. Mori, T. Kozawa, T. Ohwaki, Y. Taga, S. Nagai, S. Yamasaki, S. Asami, N. Shibata, and M. Koike, Appl. Phys. Lett. 69, 3537, (1996).

12. H. Ishikawa, S. Kobayaxhi, Y. Kiode, S. Yamasaki, S. Nagai, J. Umesaki, and M. Koike, M. Murakami, J. Appl. Phys. 81, 1315 (1997).

13. J.K. Kim, J.L. Lee, J.W. Lee, H.E. Shin, Y.J. Park, and T. Kim, Appl. Phys. Lett. 73, 2953, (1998). 